\title{
The functional relationship between visual backward masking and prepulse inhibition
}

\author{
JONATHAN K. WYNN, ${ }^{\mathrm{a}}$ MICHAEL E. DAWSON, ${ }^{\mathrm{a}}$ AND ANNE M. SCHELL ${ }^{\mathrm{b}}$ \\ ${ }^{a}$ Department of Psychology, University of Southern California, Los Angeles, California 90089, USA \\ ${ }^{\mathrm{b}}$ Department of Psychology, Occidental College, Los Angeles, California 90041, USA
}

\begin{abstract}
This experiment demonstrated a relationship between prepulse inhibition (PPI) of the startle eyeblink and visual backward masking in college students. It was hypothesized that recovery from backward masking effects is due in part to sensory gating, as assessed by auditory and visual PPI. Visual presentations of letters served as targets or visual prepulses in an intermixed session of backward masking and PPI. Backward masking and PPI (both auditory and visual) were assessed at stimulus onset asynchronies of 30, 45, 60, 120, and $150 \mathrm{~ms}$. A repeated-measures regression revealed that there was a relationship between backward masking and PPI for both visual and auditory PPI, with higher levels of PPI being associated with greater recovery from backward masking. The data are consistent with the hypothesis that recovery from backward masking effects is affected by sensory gating acting in part to gate out the interruptive effects of the mask.
\end{abstract}

Descriptors: Visual backward masking, Visual prepulse inhibition, Auditory prepulse inhibition, Information processing

Studies using paired-stimulus presentation have been invaluable for understanding how the brain is able to process information in rapid succession and how dysfunctions in that processing can be seen in pathological populations. Examples of such paradigms used to study cognitive processes in both normal populations and pathological populations, particularly patients with schizophrenia, include visual backward masking, prepulse inhibition (PPI), and P50 suppression. What is unclear, however, is whether these separate paradigms are, to some extent, indexing common mechanisms or whether they index entirely separate mechanisms.

J. K. Wynn is now at the University of California, Los Angeles. This research was conducted as part of a dissertation conducted by J. K. Wynn under the direction of M. E. Dawson. It was supported in part by NIMH grants MH46433 and MH01086 to M. E. Dawson, and additional support while preparing this article was provided by grant MH 14584 to Dr. Keith Nuechterlein. We gratefully acknowledge J. K. Wynn's committee members: Richard John, David Walsh, and Mitch Earleywine. We gratefully acknowledge the assistance of Bill Williams, Roger Dow, and Gary Thorne for programming and technical services, Susan Kesser, and Kelle Leber for help in data collection, and Michael F. Green for advice in backward masking and comments on the article. We also gratefully acknowledge the generous statistical consultation provided by Richard John, Ph.D., and Rand Wilcox, Ph.D., from the University of Southern California, and Jim Mintz, Ph.D. from University of California, Los Angeles.

Address requests for reprints to: Jonathan K. Wynn, Ph.D., VA Greater Los Angeles Healthcare System, 11301 Wilshire Blvd., MIRECC (210A), Los Angeles, CA 90073, USA. E-mail: jkwynn@ucla.edu.
Because theoretical interpretations of both prepulse inhibition and visual backward masking often refer to common neural substrates (e.g., transient and sustained systems), and both show deficits with high dopamine levels (for a review, see Braff, Saccuzzo, \& Geyer, 1991) and operate within the same time frame (approximately $30-150 \mathrm{~ms}$ ), this experiment examined the relationship between visual backward masking and prepulse inhibition.

In PPI paradigms, the startle eyeblink can be reliably and predictably modified if a nonstartling stimulus (known as a prepulse) is presented prior to a startling stimulus, even though the startle eyeblink is an automatic reflex mediated at the brainstem level (Graham, 1975; Hoffman \& Ison, 1980). More specifically, if the interval between the onset of the prepulse and the startle stimulus is relatively short (between 30 and $500 \mathrm{~ms}$ ), the startle eyeblink is inhibited, with maximal inhibition occurring around $120 \mathrm{~ms}$ (for reviews, see Blumenthal, 1999; Filion, Dawson, \& Schell, 1998). This interval is commonly referred to as the lead interval in the PPI literature. However, to avoid confusion between the different paradigms to be discussed below, we will refer to the interval between the first stimulus and the second stimulus in both PPI and masking paradigms as stimulus onset asynchrony (SOA).

PPI can be elicited by a wide range of prepulses: acoustic, visual, or tactile. In both the visual and auditory systems, neurons respond either to the transient nature or to the sustained nature of stimuli. Briefly, transient cells throughout the visual system are responsive to stimuli that have low spatial frequency 
(e.g., blurry), are sensitive to the onset, offset, and location of a stimulus, and have a short response latency. Sustained cells, however, are responsive to stimuli with a high spatial frequency (e.g., focused) and to stimuli that are continuous, and have a longer response latency (see review by Breitmeyer \& Ganz, 1976, pp. 10-13). Similar systems of transient and sustained cells have been found throughout the auditory system (Gersuni, 1971; for a review, see Pickles, 1988) with transient neurons responding to stimulus characteristics such as onset and offset and sustained neurons responding to the prolonged stimulus output.

Graham (1975) has hypothesized that PPI is initiated by the transient portion of the prepulse (i.e., the onset or offset of the prepulse) whereas the sustained portion of the prepulse (i.e., the continuous portion between the onset and offset) can attenuate the inhibition. Graham and Murray (1977) have hypothesized that acoustic elicitation of startle and prepulse inhibition are controlled by the activation of short time-constant (e.g., onsets, offsets), transient-sensitive neurons. Several studies have supported the hypothesis that the transient system makes the greatest contribution to PPI, at least if the prepulse is in the auditory modality (Blumenthal \& Levey, 1989; Graham \& Murray, 1977; Lane, Ornitz, \& Guthrie, 1991; Wynn, Dawson, \& Schell, 2000). More recently, it has been shown than the transient system in the visual modality also makes the greatest contribution to PPI (Wynn, Dawson, \& Schell, 2001).

Prepulse inhibition is thought to reflect an automatic sensorimotor gating mechanism that serves to protect the processing of the prepulse (e.g., Graham, 1992). According to this hypothesis, the prepulse sets up a negative feedback loop (Graham, 1975) that inhibits processing of and response to a subsequent startle pulse and prevents that startle from interfering with processing of the prepulse. Thus, the amount of prepulse inhibition exhibited reflects how well the prepulse was processed. Greater PPI reflects stronger processing of the prepulse as it was protected from interruption by the startle pulse.

Visual backward masking refers to the phenomenon in which the processing of a visual stimulus (a target) is interrupted by the presentation of another visual stimulus (a mask) which follows the target very quickly, at intervals of approximately 10-300 ms. Breitmeyer and Ganz (1976) have proposed that transient stimulation resulting from the presentation of the mask laterally inhibits the processing by sustained channels of the target, thus interrupting processing of the target. In a recent update to this influential model, Breitmeyer and Ogmen (2000) have extended the sustained-transient model to incorporate transient-onsustained effects that occur in postretinal areas. The model proposes that transient neural activation can interrupt the sustained oscillations (in the $40-\mathrm{Hz}$ gamma range) in the cortical sustained pathway. If the temporal separation of the target and the mask is either too short or too long for the activation of the transient channels by the presentation of the mask to affect the sustained processing of the target, masking effects are decreased or nonexistent (Breitmeyer, 1980).

Green, Nuechterlein, and Mintz (1994), in studying backward masking abnormalities (i.e., requiring more time to recover from backward masking) in schizophrenia, found that patients showed the most abnormalities in masking paradigms that isolated the transient channels. They further comment that "backward masking might be viewed as a special condition of the general phenomenon of sensory gating" (p. 949) and that the backward masking deficits exhibited by schizophrenia patients may be due to the failure to gate out the disruptive effects of the mask. Furthermore, Braff (1993) also suggests that visual backward masking and prepulse inhibition may have a "common neural substrate" (p. 246). Due to the similar nature of the time course of the development of PPI and the recovery from backward masking effects, it is possible, as Green et al. and Braff suggest, that backward masking may be reflecting sensory gating to some extent.

Interestingly, only one study to date has measured backward masking and prepulse inhibition from the same subjects in the same experimental session. While not directly correlating backward masking to prepulse inhibition, Perry and Braff (1994) concluded that both of these variables were related to measures of thought disorder (derived from a Rorschach-based Ego Impairment Index) in schizophrenia patients. To our knowledge, this is the closest that investigators have come to relating backward masking to prepulse inhibition.

The present experiment attempted to determine the association between backward masking and sensory gating, as assessed by PPI, by measuring both backward masking and PPI in the same testing session. It was hypothesized that both recovery from backward masking and PPI reflect a common sensory gating mechanism. That is, the recovery from the effects of the mask on the target is due at least in part to a sensory gating mechanism becoming fully active to gate out the stimulus energy of the mask, thus reducing the neural response to its presence.

It was hypothesized that recovery from masking is facilitated when the gating mechanism initiated by the target (which served as a visual prepulse) has developed fully enough to gate out the interrupting effects of the mask. If reduced effects of the mask at later SOAs are related to sensory gating, a significant relationship should be seen between the number of correct detections of the target (i.e., reduced masking effects resulting in a greater number of correct hits) and the amount of PPI. We hypothesized that PPI would be correlated with reduced backward masking effects, consistent with the hypothesis that sensory gating may be decreasing the effectiveness of the mask, thus reducing masking effects.

In addition, we hypothesized that auditory and visual PPI would be positively correlated across all subjects, providing further support for a common sensory gating mechanism. The assessment of PPI using both auditory and visual prepulses in the same session and employing the same startle stimulus allowed for the investigation of this relationship.

\section{Methods}

\section{Participants}

Participants were drawn from undergraduate psychology classes at the University of Southern California. Participants' rights were guaranteed by the University of Southern California Institutional Review Board. Data from a total of 52 participants were collected, 37 women and 15 men. After exclusions (see Results) there were a total of 36 participants, 11 men and 25 women.

\section{Experimental Stimuli}

Auditory prepulses and visual prepulses (letters presented alone without the mask) were $15 \mathrm{~ms}$ in duration. Pilot data showed that $15 \mathrm{~ms}$ was ample time to correctly detect the target presented alone. Auditory prepulses were $75 \mathrm{~dB}(\mathrm{~A})$ SPL white noise bursts 
with near instantaneous rise/fall times. The startle stimulus consisted of a 50-ms, near instantaneous rise/fall time, $109 \mathrm{~dB}(\mathrm{~A})$ SPL white noise burst. Auditory stimuli were presented through binaural Telephonics TDH-50P headphones. Decibel levels were recorded with a Realistic sound level meter using a Quest Electronics earphone coupler.

Visual stimuli were presented from two Kodak Carousel 4600 slide projectors, stacked on top of each other using a Pacific Innovations slide projector stacking system, housed in a soundproof chamber situated behind the participant. Tachistoscopic shutters were used on the slide projectors, interfaced with the computer to control timing of presentation. Visual stimuli were presented on the wall in front of the participant. The distance from the wall to the participant was approximately $1.4 \mathrm{~m}$. The illuminance of the visual stimuli, as measured by placing an Extech light meter directly on the wall area receiving the stimuli, was 250.8 lux.

\section{Recording and Scoring of Dependent Variables}

Eyeblinks were scored as EMG activity of the orbicularis oculi muscle of the left eye. One small $(4 \mathrm{~mm})$ silver-silver chloride electrode was placed on the left lower eyelid directly below the pupil and a second 4-mm electrode was placed approximately $1 \mathrm{~cm}$ lateral to the first (measuring from the edges of the electrode). The impedance between the two electrodes was measured and was deemed acceptable below $10 \mathrm{k} \Omega$. Stimulus presentation and data acquisition were controlled through Contact Precision Instruments equipment and a computer running Psylab 7 software. The raw EMG signal (filtered at $10 \mathrm{~Hz}$ high pass [12 dB/octave] and $500 \mathrm{~Hz}$ low pass) was collected at a rate of $1,000 \mathrm{~Hz}$. The data were stored and exported for analysis in microvolts. For analysis, the raw EMG signal was rectified and then software integrated using a custom-written scoring program that simulates an integrator circuit with a $20-\mathrm{ms}$ time constant. Startle response onset was set to be detected within a window of $20-120 \mathrm{~ms}$ whereas peak activity was set within a window of 30-200 ms. Peak amplitude is the difference between the peak and baseline, where baseline is the average of the $20 \mathrm{~ms}$ of EMG activity immediately following startle pulse onset.

Prepulse inhibition was calculated as a percent change score: [(prepulsed startle - startle alone $) /($ startle alone $) \times 100]$. Percent change units are preferred over difference scores (prepulsed startle-startle alone) because difference scores in absolute microvolt units are correlated with startle alone blink amplitude whereas percent change scores are not, removing any dependence on unmodified startle amplitude (Jennings, Schell, Filion, \& Dawson, 1996). Effects of backward masking were assessed by determining the number of correct hits at each SOA (ranging from 0 to 10 for each target-to-mask SOA and target alone).

\section{Procedure}

Upon the participant's arrival to the laboratory, electrodes were attached for recording of electromyographic (EMG) responses. Participants were instructed that they would see one of 15 letters (A, E, F, H, I, K, L, M, N, T, V, W, X, Y, and Z) presented very briefly on the wall in front of them. These letters served on separate trials as targets in the backward masking task and as visual prepulses to assess PPI. Participants were told that they were to try to name the letter that they were shown as quickly as possible. They were also warned that on some occasions, another slide with a random pattern of lines would be shown shortly after the slide with the letter and that this slide could be ignored. If they did not clearly see the letter or were unsure of the letter, they were to choose one of the 15 letters that were typed on a list and posted in front of the participant. The experimenter, sitting in an adjoining room and monitoring the participant's responses through a speaker, recorded the participant's response after each trial.

Participants were also told that they would hear a lowintensity, brief staticlike noise on certain trials (this served as the auditory prepulse). They were instructed to say "heard" whenever they heard this noise. Furthermore, participants were instructed that they would hear a much louder staticlike noise (serving as the startle stimulus) and that this noise could simply be ignored. To familiarize the participants with the low-intensity and high-intensity noises, two examples of each were presented.

To ensure that participants remained motivated to perform the task, they were offered a bonus of $\$ 5$. However, participants were told that for each incorrect letter identified, $\$ 0.10$ would be subtracted from their bonus.

For the experimental session, 100 trials were presented: 10 visual target alone trials, 50 masking trials (10 at each SOAs of $30,45,60,120$, and $150 \mathrm{~ms}$ ), 20 visual PPI trials (4 at each SOA between the visual target stimulus and the startle stimulus; masks were not presented on these trials), and 20 auditory PPI trials (4 at each SOA between the auditory prepulse and the startle stimulus). The visual mask was not presented during auditory PPI trials. There were also a total of 20 intertrial interval (ITI) startle-alone white noise probes that served to indicate the participants' baseline startle responding. Responses to these 20 ITI probes were averaged to create a startle-alone baseline for each participant. A total of 60 startle probes were presented: 20 ITI startle-alone probes and 4 startle probes at each of the 5 SOAs following the auditory and visual prepulses. ITI length averaged $11 \mathrm{~s}$ (range 10-12 s) with ITI startle probes presented an average of $6 \mathrm{~s}$ (range 5-7s) into the trial. All stimuli were presented in a randomized order that was fixed for every participant.

The entire experimental testing session lasted approximately $25 \mathrm{~min}$. Halfway through the experiment, participants were informed how much time they had left so as to keep the participants alert and focused on their task. At the end of the experiment participants were told how many correct letters they identified and were paid their bonus.

\section{Statistical Analysis and Design}

Two independent variables were manipulated within subjects to assess auditory and visual PPI. The first independent variable was modality, with prepulses in either the visual or auditory modality. The second independent variable was SOA, with SOAs of $30,45,60,120$, and $150 \mathrm{~ms}$. Visual backward masking was assessed using a one-way repeated-measures design, with SOA as the independent variable. Six SOAs were used: $0 \mathrm{~ms}$ (no mask), $30,45,60,120$, and $150 \mathrm{~ms}$.

To assess whether prepulse inhibition is associated with recovery from backward masking effects, a repeated-measures regression model with two time-dependent predictors (PPI and SOA) was used to test the hypothesis that PPI is a predictor of recovery from backward masking. Separate equations were used to assess this relationship for auditory and visual prepulse inhibition. As the relationship between recovery from backward masking and PPI was the only hypothesis to be tested, a significant value for the within-subjects slope of the regression of backward masking on PPI had to be shown to reject the null 
hypothesis. The significance test determines whether or not there is a significant relationship between recovery and PPI. Both PPI and SOA were added as predictors to the equation. As both backward masking and PPI are time-dependent variables, it was necessary to add SOA as a predictor. If the slope for PPI as a predictor remains significant in this equation with SOA as a predictor, PPI is accounting for a significant amount of the variance in backward masking independently of that attributed to the mutual dependence of both backward masking and PPI on SOA.

\section{Results}

\section{Exclusions and Outlier Analysis}

Before any analysis, any participant scoring less than an average of $2.0 \mu \mathrm{V}$ peak amplitude for his/her eyeblink response to startlealone trials (based on the average of all 20 ITI startle-alone trials) was considered a nonresponder and was excluded from analysis. Furthermore, any participant scoring more than two errors on the letter-naming task when the letter was presented alone was excluded from analysis due to an inability to perform the task adequately. A total of 16 participants met one of these criteria (11 with an average ITI less than $2.0 \mu \mathrm{V}, 2$ with more than two misses to the target presented alone, and 3 with a combination of both). This left a total of 36 participants (11 men, 25 women). After exclusions, the average ITI startle amplitude was $12.34 \mu \mathrm{V}$ $(S D=12.15 \mu \mathrm{V})$.

After these exclusions, outlier analyses were conducted on the EMG values to the prepulse + startle trials, to startle-alone trials, and on percent change scores. An outlier was considered to be any score more than three standard deviations from the mean and more than two standard deviations from the next highest or lowest score. Based on these rules, there were no outliers among the data. For all analyses Greenhouse-Geisser $(\varepsilon)$ corrections were used for repeated-measures analyses of variances with more than one degree of freedom. We report uncorrected degrees of freedom, corrected $p$ values, and $\varepsilon$ values. Rom's procedure (Rom, 1990) was used for all groups of post hoc $t$ tests to control for experiment-wise Type I Error.

\section{Backward Masking Analysis}

A one-way repeated measures ANOVA was used to assess the effect of SOA on backward masking. Accuracy of identification of the target presented alone (SOA-0 ms: mean 9.69, SD 0.62; a perfect score was 10) and for the other five SOA conditions were used as the repeated measure. Means are shown in Figure 1 (note that in Figure 1, the scores have been converted to percent correct to bring them in scale with the PPI scores for visual comparison only).

A main effect of SOA was shown, $F(5,175)=225.754$, $p<.001, \varepsilon=.575$. The number of correct hits was minimal when the target was followed by a mask at an SOA of $30 \mathrm{~ms}$. From 30 to $150 \mathrm{~ms}$, the number of correct hits for the target increased, with asymptote being reached at $120 \mathrm{~ms}$. Compared to the target presented alone, post hoc analysis of SOAs of 30, 45, and $60 \mathrm{~ms}$ revealed significantly fewer correct hits, all $t \mathrm{~s}(35)>10.454$, $p<.001$.

\section{Auditory PPI Analysis}

Percent change startle modification scores for auditory prepulses were analyzed with a one-way repeated measures ANOVA. The five SOAs were used as the repeated measure. Results showed a

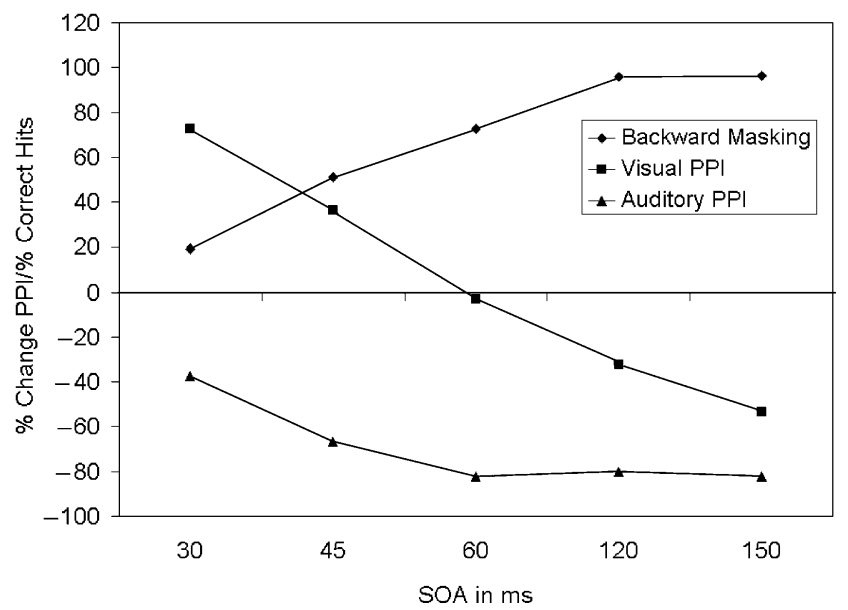

Figure 1. Mean percent change in PPI scores, for both auditory and visual PPI, and the percent of correct hits in the backward masking trials at each SOA. Note that hit rate for backward masking was converted to a percent correct to bring those values into scale with the PPI scores.

main effect of SOA, $F(4,140)=41.168, p<.001, \varepsilon=.661$. As can be seen in Figure 1, PPI increased as the SOA increased from 30 to $60 \mathrm{~ms}$ and leveled off from 60 to $150 \mathrm{~ms}$. Post hoc analyses revealed that all auditory PPI scores were significantly different from zero, all $t \mathrm{~s}(35)<-7.983, p<.001$.

\section{Visual PPI Analysis}

Percent change startle modification scores for visual prepulses were analyzed with a one-way repeated measures ANOVA. The five SOAs were used as the repeated measure. Results showed a main effect of SOA, $F(4,140)=51.765, p<.001, \varepsilon=.439$. As can be seen in Figure 1, percent change PPI scores steadily changed from facilitation to increasing inhibition from an SOA of $30 \mathrm{~ms}$ to an SOA of $150 \mathrm{~ms}$. However, the post hoc analysis of visual PPI scores revealed that only SOAs of 120 and $150 \mathrm{~ms}$ produced significant amounts of PPI, $t$ s $(35)<-4.626, p<.001$.

\section{Regression Analysis of Visual PPI and Recovery from Backward Masking}

When SOA was entered as a predictor of backward masking, results showed that the mean of the within-subject slopes for the regression of recovery from backward masking on SOA was significantly positive, $t(35)=11.625, p<.001$, indicating a significant positive relationship between recovery from backward masking and SOA, consistent with the ANOVA results. The mean of the slopes for the regression of recovery from backward masking on visual PPI was also significant, $t(35)=-2.564$, $p<.02$, indicating a significant negative relationship between backward masking recovery and visual PPI. The percentage of the variance in backward masking accounted for by PPI and SOA together was $70.2 \%$. Most important, a significant relationship was seen between visual PPI and recovery from backward masking effects even when the contribution of SOA to recovery is included in the equation. That is, PPI predicts recovery from backward masking independently of their mutual relationship with SOA.

\section{Regression Analysis of Auditory PPI and Recovery from Backward Masking}

When SOA was entered as a predictor in this equation, the mean of the slopes for the regression of recovery from backward 
masking on SOA was significantly positive, $t(35)=12.992$, $p<.001$, indicating a significant positive relationship between recovery from backward masking and SOA. The mean of the slopes for the regression of backward masking recovery on PPI was also significant, $t(35)=-6.098, p<.001$, indicating a significant negative relationship between recovery from backward masking and auditory PPI. The percentage of the variance in backward masking accounted for by PPI and SOA together was $77.4 \%$. As with the visual PPI results, a significant relationship remained between auditory PPI and recovery from backward masking even when the contribution of SOA to recovery is included in the equation.

\section{Correlations between Auditory PPI and Visual PPI}

To determine whether visual PPI and auditory PPI were related, the average amount of PPI produced by visual prepulses across all SOAs was correlated with the average amount of PPI produced by auditory prepulses across all SOAs. A significant positive relationship was seen, Pearson's $r(34)=.378, p<.05$, reflecting that as the amount of visual PPI increased the amount of auditory PPI also increased.

\section{Discussion}

Based on an influential model of backward masking developed by Breitmeyer and Ganz (1976), and updated by Breitmeyer and Ogmen (2000), backward masking effects are reduced when sufficient time (i.e., SOA) has passed so that the transient channel activity of the mask no longer overlaps with and interrupts processing of the high-spatial frequency sustained channel activity of the target (Breitmeyer, 1980). This model views the reduction of masking at longer SOAs as simply reflecting greater time allowed for sustained channel neurons to complete processing. The results of the present study suggest an additional time-dependent mechanism contributing to recovery from masking: the development of sensory gating initiated by the target, which acts to screen out the stimulus energy of the mask, thus reducing the transient system activity that it produces. As both auditory and visual PPI are related to recovery from backward masking effects, with greater PPI being associated with faster recovery (greater hit rate), the results suggest that the transient onset of the target acts as a prepulse, further reducing the interruptive effects of the mask. The transient-sustained model of masking has recently been updated and expanded, and other models of masking such as the Perceptual Retouch model and Boundary Contour System model have been proposed (see Breitmeyer and Ogmen, 2000, for a thorough review). However, it is clear that in any model, any process that reduces the neural response to the mask or its overlap with the response to the target (e.g., longer SOAs, and possibly gating out of the mask) will result in less backward masking. These results could help explain why schizophrenia patients are deficient in both PPI and visual backward masking: These patients may have a defective sensory gating mechanism that affects both visual backward masking and prepulse inhibition.

Prepulse inhibition was a significant predictor of recovery from backward masking effects (i.e., greater hit rate) over and above the predictive value of SOA. This was true for both auditory and visual PPI. If the relationship between PPI and recovery from masking were due purely to a mutual dependence of PPI and recovery from backward masking on SOA, adding $\mathrm{SOA}$ as a predictor in the equation would account for all of the shared variance and PPI would no longer show a separate significant relationship to recovery from backward masking. In essence, SOA would entirely explain the relationship between PPI and recovery from backward masking. However, even accounting for SOA, a significant relationship remained between both auditory and visual PPI and recovery from backward masking. Higher levels of PPI (more negative values) were associated with greater recovery from backward masking.

The inference of overlapping sensory gating mechanism components is supported by the cross-modal relationship seen between recovery from backward masking and auditory PPI, and by the significant positive correlation between auditory and visual PPI. If there were a separate gating mechanism initiated by visual stimuli and auditory stimuli, there would be no relationship between auditory PPI and recovery from visual backward masking. However, as the results have shown, this is not the case. By using the same visual stimulus that served as the backward masking target as the visual prepulse, it is clear that whatever form of processing occurs of this stimulus has to be occurring when both of the second stimuli, either the mask or the startle pulse, are presented.

The hypothesis of a common sensory gating mechanism is made all the more strong by the positive correlation between auditory and visual PPI. Though auditory and visual PPI have been studied extensively, it is believed that this is only the second study to show a direct relationship between the two in the same participants and in the same testing session (the first being Wynn et al., 2001). Although auditory and visual PPI are correlated, they clearly follow different time courses, as seen in Figure 1. This may likely be due to the physical properties of the neural mechanisms responding to the different stimuli. It may take longer for visual PPI to develop because there are electrochemical reactions that must occur in the retina before those signals are passed onward for further processing. Although the time courses may be different, it is highly unlikely auditory and visual PPI are indexing entirely different processes. As SOA increased, visual PPI was converging on the maximum amount of PPI seen to the auditory prepulse.

The results of this study are consistent with and help to explain the findings that schizophrenia patients are deficient in both PPI and backward masking. It has been shown that schizophrenia patients show deficient PPI as compared to normal controls, reflecting poor or defective sensorimotor gating (Braff et al., 1978; Braff, Grillon, \& Geyer, 1992). Furthermore, schizophrenia patients show slower recovery from backward masking effects (Green et al., 1994; Saccuzzo, Hirt, \& Spencer, 1974). The abnormalities in both the backward masking and PPI in schizophrenia suggest that schizophrenia patients have a sensory gating dysfunction.

Whereas this study examined the relationship between masking and prepulse inhibition, other studies have attempted to relate other paired-stimulus paradigms (e.g., P50 suppression) to prepulse inhibition. The results of this endeavor have proved to be mixed at best. Whereas some have shown a modest correlation between P50 suppression and PPI measured in separate sessions (e.g., Oranje et al., 1999), others have shown no relationship between P50 suppression and PPI while recording in the same session (e.g., Light \& Braff, 2001).

Though the results of this article are promising, there are a number of caveats that must be discussed. First, these results cannot speak to direct neurophysiological links between visual backward masking and prepulse inhibition. As this was a 
correlational design, we are not able to make strong inferences about any common underlying sensory gating mechanism for recovery from masking and PPI. However, these results are consistent with the hypothesis that sensory gating that is in effect at later SOAs contributes to making the mask less effective, thus leading to reduced backward masking effects. Another caveat is that by measuring both PPI and backward masking we may have altered the effects that would normally be seen when measuring these tasks separately. However, we tried to control for this effect by having at least the same task performed for the visual stimuli in both the separate masking and prepulse inhibition trials. In addition, on visual inspection of the functions seen in Figure 1 relating SOA to backward masking and amount of auditory and visual PPI are comparable to those seen in other studies.

The quantitative differences in auditory and visual PPI should also be mentioned. Visual and auditory prepulses most likely differed in subjective intensity, contributing to greater overall PPI for auditory prepulses. As noted by Blumenthal (1999), intensity influences the amount of PPI seen. Furthermore, at the earliest SOAs, visual prepulses resulted in prepulse facilitation. Facilitation at early SOAs may be due to a different underlying mechanism than inhibition (e.g., Sarno, Blumenthal, \& Boelhouwer, 1997; Schmolesky, Boelhouwer, \& Blumenthal, 1996). However, it is clear that the visual prepulse is inducing inhibition, as at each successive SOA the amount of startle is being decreased and at the two latest SOAs (120 and $150 \mathrm{~ms}$ ), there is, in fact, significant inhibition of startle. It is in this SOA range where both maximum startle inhibition and little to no masking effects are seen.

Though it appears that transient channels may be implicated in the production of both prepulse inhibition and reduction of backward masking effects, it is unclear whether overactive or underactive transient channels are responsible for the abnormalities in these processes seen in schizophrenia patients. Most research in backward masking in schizophrenia has theorized that overactive transient channels are responsible for their abnormal masking results. In theory, overactive transient channels could also result in deficient PPI as the transient onset of the startle may be much more powerful than in normals and overcome any gating that may have been initiated by the prepulse. In backward masking, overactive transient channels would lead to much more effective masking and longer masking effects as the transient onset of the mask would be much more persistent. This conclusion has been reached by Green et al. (1994). The exact nature of overactive or underactive transient channels in schizophrenia patients obviously requires much more empirical research to fully understand the nature of their deficits in prepulse inhibition and backward masking.

In conclusion, the results are promising in showing that recovery from backward masking and prepulse inhibition are associated and may be influenced by common sensory gating mechanisms. These results have several implications for normal functioning and for those who are afflicted with informationprocessing deficits, and more specifically for schizophrenia patients.

\section{REFERENCES}

Blumenthal, T. D. (1999). Short lead interval startle modification. In M. E. Dawson, A. M. Schell, \& A. H. Böhmelt (Eds.), Startle modification: Implications for neuroscience, cognitive science, and clinical science (pp. 51-71). New York: Cambridge University Press.

Blumenthal, T. D., \& Levey, B. J. (1989). Prepulse rise time and startle reflex modification: Different effects for discrete and continuous prepulses. Psychophysiology, 26, 158-165.

Braff, D. L. (1993). Information processing and attention dysfunctions in schizophrenia. Schizophrenia Bulletin, 19, 233-259.

Braff, D. L., Grillon, C., \& Geyer, M. A. (1992). Gating and habituation of the startle reflex in schizophrenic patients. Archives of General Psychiatry, 49, 206-215.

Braff, D. L., Saccuzzo, D. P., \& Geyer, M. A. (1991). Information processing dysfunctions in schizophrenia: Studies of visual backward masking, sensorimotor gating, and habituation. In S. R. Steinhauer, J. H. Gruzelier, \& J. Zubin (Eds.), Handbook of schizophrenia, Vol. 5: Neuropsychology, psychophysiology, and information processing (pp. 303-334). Amsterdam: Elsevier Science Publishers.

Braff, D., Stone, C., Callaway, E., Geyer, M., Glick, I., \& Bali, L. (1978). Prestimulus effects on human startle reflex in normals and schizophrenics. Psychophysiology, 15, 339-343.

Breitmeyer, B. G. (1980). Unmasking visual masking: A look at the "why" behind the veil of the "how." Psychological Review, 87, 52-69.

Breitmeyer, B. G., \& Ganz, L. (1976). Implications of sustained and transient channels for theories of visual pattern masking, saccadic suppression, and information processing. Psychological Review, 83, $1-36$.

Breitmeyer, B. G., \& Ogmen, H. (2000). Recent models and findings in visual backward masking: A comparison, review, and update. Perception and Psychophysics, 62, 1572-1595.

Filion, D. L., Dawson, M. E., \& Schell, A. M. (1998). The psychological significance of human startle eyeblink modification: A review. Biological Psychology, 47, 1-43.

Gersuni, G. V. (1971). Temporal organization of the auditory function. In G. V. Gersuni (Ed.), Sensory processes at the neuronal and behavioral levels (pp. 85-114). New York: Academic Press.

Graham, F. K. (1975). The more or less startling effects of weak prestimulation. Psychophysiology, 12, 238-248.
Graham, F. K. (1992). Attention: The heartbeat, the blink, and the brain. In B. A. Campbell, H. Hayne, \& R. Richardson (Eds.), Attention and information processing in infants and adults: Perspectives from human and animal research. Hillsdale, NJ: Lawrence Erlbaum Associates.

Graham, F. K., \& Murray, G. M. (1977). Discordant effects of weak prestimulation on magnitude and latency of the reflex blink. Physiological Psychology, 5, 108-114.

Green, M. F., Nuechterlein, K. H., \& Mintz, J. (1994). Backward masking in schizophrenia and mania. II. Specifying the visual channels. Archives of General Psychiatry, 51, 945-951.

Hoffman, H. S., \& Ison, J. R. (1980). Reflex modification in the domain of startle: 1. Some empirical findings and their implications for how the nervous system processes sensory input. Psychological Review, 87 , 175-189.

Jennings, P. D., Schell, A. M., Filion, D. L., \& Dawson, M. E. (1996). Tracking early and late stages of information processing: Contributions of startle eyeblink reflex modification. Psychophysiology, 33, $148-155$.

Lane, S. J., Ornitz, E. M., \& Guthrie, D. (1991). Modulatory influence of continuous tone, tone offset, and tone onset on the human acoustic startle response. Psychophysiology, 28, 579-587.

Light, G. A., \& Braff, D. L. (2001). Measuring P50 suppression and prepulse inhibition in a single recording session. American Journal of Psychiatry, 158, 2066-2068.

Oranje, B., van Berckel, B. N. M., Kemner, C., van Ree, J. M., Kahn, R. S., \& Verbaten, M. N. (1999). P50 suppression and prepulse inhibition of the startle reflex in humans: A correlational study. Biological Psychiatry, 45, 883-890.

Perry, W., \& Braff, D. L. (1994). Information-processing deficits and thought disorder in schizophrenia. American Journal of Psychiatry, $151,363-367$.

Pickles, J. O. (1988). An introduction to the physiology of hearing (2nd ed). London: Academic Press.

Rom, D. M. (1990). A sequentially rejective test procedure based on a modified Bonferroni inequality. Biometrika, 77, 663-666.

Saccuzzo, D. P., Hirt, M., \& Spencer, T. J. (1974). Backward masking as a measure of attention in schizophrenia. Journal of Abnormal Psychology, 83, 512-522. 
Sarno, A. J., Blumenthal, T. D., \& Boelhouwer, A. J. W. (1997). Modification of the electrically elicited eyeblink by acoustic, visual, and vibrotactile pulses. Psychobiology, 25, 253-265.

Schmolesky, M. T., Boelhouwer, A. J. W., \& Blumenthal, T. D. (1996). The effect of acoustic pulse intensity upon the electrically elicited blink reflex at positive and negative stimulus onset asynchronies. Biological Psychology, 44, 69-84

Wynn, J. K., Dawson, M. E., \& Schell, A. M. (2000). Discrete and continuous prepulses have differential effects on startle prepulse inhibition and skin conductance orienting. Psychophysiology, 37, 224-230.

Wynn, J. K., Dawson, M. E., \& Schell, A. M. (2001). Effects of attention and modality on prepulse inhibition and skin conductance responding using discrete and continuous prepulses. Psycholphysiology, 38 (Suppl. 1), S102.

(ReCeIved February 13, 2003; ACCEPTed August 8, 2003) 